\title{
Random measures and harmonizable sequences
}

by

\section{K. U R B A I K (Wroclaw)}

By a harmonizable sequence of random variables we mean a sequence of Fourier coefficients of a random measure. A concept of prediction for strictly stationary sequences which need not have a finite variance was introduced in [19] and [21]. In particular, each stationary sequence admitting a prediction is the sum of two independent stationary sequences, one deterministic and the other completely non-deterministic. The purpose of this paper is to give a characterization of deterministic and completely non-deterministic harmonizable stationary sequences of random variables. Some modular spaces introduced by J. Musielak and W. Orlicz in [14] are used as a tool to study harmonizable sequences. They play the same role in our investigations as the $L^{2}$-spaces in the Wiener-Kolmogorov theory of the best linear least squares prediction for wide sense stationary sequences.

The first section contains a discussion of an extremal problem for Musielak-Orlicz spaces and a generalization of the famous Kolmogorov-Krein criterion for $L^{p}$-spaces. The second one contains an analogue of S. Bernstein's Theorem concerning Gaussian random variables. In the third section we study the space of all complex-valued functions which are integrable with respect to a complex-valued isotropic random measure. The main results concerning harmonizable sequences are given in the last section.

1. An extremal problem for Musielak-Orlicz spaces. Given a measure $\nu$ defined on Borel subsets of the unit interval $I=[0,1]$, we take a real function $\Phi$ defined on $I \times R_{+}, R_{+}$being the space of non-negative reals, satisfying the following conditions:

(i) $\Phi(t, 0)=0$ and $\Phi(t, x)>0$ for $x>0$ and $\nu$-almost all $t$;

(ii) $\Phi(t, x)$ is a continuous non-decreasing function of $x$ for every $t \epsilon I$;

(iii) $\Phi(t, x)$ is. Borel measurable as a function of $t$ for every $x \in R_{+}$;

(iv) $\int_{I} \Phi(t, 1) v(d t)<\infty$ 
(v) (the $\Delta_{2}$-condition) there exists a positive constant $x$ such that

$$
\Phi(t, 2 x) \leqslant x \Phi(t, x)
$$

for all $x$ and $v$-almost all $t$.

Throughout this paper we identify functions equal $v$-almost everywhere. Let $f$ be a complex-valued Borel function on $I$. It is easily seen that $\Phi(t,|f(t)|)$ is also a Borel function on $I$. We define a modular $\varrho$ by means of the formula

$$
\varrho(f)=\int_{I} \Phi(t,|f(t)|) v(d t) .
$$

Let $L_{\tilde{\phi}}(\nu)$ be the set of all complex-valued Borel functions $f$ on $I$ such that $\varrho(f)$ is finite. The set $L_{\phi}(\nu)$ is a linear space over the complex field under usual addition and scalar multiplication. Moreover, it becomes a complete linear metric space under the non-homogeneous norm

$$
\|f\|=\inf \left\{c: c>0, \varrho\left(c^{-1} f\right) \leqslant c\right\} .
$$

The space $L_{\mathscr{D}}(\nu)$ with this norm was introduced and investigated by J. Musielak and W. Orlicz in [14] and will be called a Musielak-Orlice space.

A sequence $\left\{f_{n}\right\}$ of elements of $L_{\Phi}(v)$ is said to be modular convergent to an element $f$ of $L_{\Phi}(v)$ if

$$
\lim _{n \rightarrow \infty} \varrho\left(f_{n}-f\right)=0 .
$$

From the $\Delta_{2}$-condition it follows that the modular convergence is equivalent with the norm convergence in $L_{\Phi}(v)$ (see [14], theorem 1.31). Further, from (iv) it follows that all bounded Borel functions belong to $L_{\Phi}(v)$. Moreover, the set of all Borel simple functions, i.e. Borel functions assuming a finite number of values, is dense in $I_{\Phi}(\nu)$.

By $v_{c}$ we shall denote the absolutely continuous component of the measure $v$ and by $d v_{c} / d t$ a Borel measurable version of its Radon-Nikodym density function. It is clear that if the Lebesgue measure is absolutely continuous with respect to the measure $v$, then

$$
\frac{d v_{c}}{d t}=\left(\frac{d t}{d v}\right)^{-1}
$$

almost everywhere in the sense of the Lebesgue measure.

We introduce auxiliary functions $\Lambda_{\varphi, v}$ and $\Omega_{\varphi, v, n}(n=1,2, \ldots)$ by means of the formulas

$$
\begin{aligned}
& \Lambda_{\Phi, v}(t, x)=\sup \left\{\frac{\log y}{\Phi(t, y)}\left(\frac{d v_{c}}{d t}\right)^{-1}: y \geqslant x\right\}, \\
& \Omega_{\Phi, v, n}(t)=\inf \left\{x: \Lambda_{\varphi, v}(t, x) \leqslant n, x \geqslant 1\right\},
\end{aligned}
$$

where the infimum of an empty set is defined as $\infty$. It is clear that all these functions are Borel measurable and

$$
1 \leqslant \Omega_{\Phi, v, n}(t) \leqslant \infty \quad(n=1,2, \ldots) .
$$

The aim of this section is to prove the following theorem:

THEOREM 1.1. Let $L_{\Phi}(v)$ be a. Musielak-Orlicz space with the norm \| \|. The equation

$$
\inf \left\|1+\sum_{k=1}^{n} a_{k} e^{2 \pi i k t}\right\|=0
$$

where the infimum is taken over all complex numbers $a_{1}, a_{2}, \ldots, a_{n}$ and $n=1,2, \ldots$, holds if and onty if no function $\log \Omega_{\Phi, v, n}(n=1,2, \ldots)$ is Lebesgue integrable over $I$.

This solution of an extremal problem of Szegö's type can be regarded as a generalization of the Kolmogorov-Krein criterion for $L^{p}$-spaces (see [7] and [8]). For a class of Orlicz spaces more general problem was discussed in $[20]$. Before proving the theorem we shall prove four lemmas.

Given an arbitrary set $\mathscr{S}$ of complex-valued Borel functions on $I$, we put $i(\mathscr{S})=\inf \{\varrho(f): f \epsilon \mathscr{P}\}$, where $\varrho$ is the modular in $L_{\Phi}(v)$. Let $\mathscr{P}$ be the set of all trigonometric polynomials

$$
1+\sum_{k=1}^{n} a_{k} e^{2 \pi i k t},
$$

where $a_{1}, a_{2}, \ldots, a_{n}$ are complex numbers and $n$ is variable. Further, let 2 be the set of all Borel functions on $I$ such that $\log |f|$ is Lebesgue integrable and $\int_{I} \log |f(t)| d t \geqslant 0$.

LEMMA 1.1. $i(\mathscr{P})=i(\mathscr{Q})$.

Proof. The inclusion $\mathscr{P} \subset \mathscr{Q}$ is a simple consequence of the Jensen inequality. Hence we get the inequality $i(\mathscr{P}) \geqslant i(\mathscr{Q})$

To prove the converse inequality we put

$$
\mathscr{Q}_{a, b}=\{f: a<|f|<b\} \cap \mathscr{Q} \quad(a, b>0) .
$$

By bounded convergence theorem we get the formula

$$
i(2)=\lim _{\substack{a \rightarrow 0 \\ b \rightarrow \infty}} i\left(\mathscr{Q}_{a, b}\right) .
$$

Consider an auxiliary modular

$$
\varrho_{0}(f)=\varrho(f)+\int_{I}|f(t)| d t
$$


on $\mathscr{Q}_{a, b}$. From condition (iv) it follows that the subset of $\mathscr{Q}_{a, b}$ consisting of trigonometric polynomials

$$
\sum_{k=-m}^{m} b_{l_{l}} e^{2 \pi i k t}
$$

where $b_{-m}, b_{-m+1}, \ldots, b_{m}$ are complex numbers and $m$ is variable, is dense in $\mathscr{Q}_{a, b}$ in the sense of the modular $\varrho_{0}$-convergence. Moreover, both functionals $\varrho(f)$ and $\int_{I} \log |f(t)| d t$ are continuous on $\mathscr{Q}_{a, b}$. Consequently, by (1.6), for every positive number $\varepsilon$ there exists a trigonometric polynomial

$$
g(t)=e^{-2 \pi i m t}\left(\sum_{k=0}^{n} c_{k} e^{2 \pi i k t}\right) \quad(m, n \geqslant 0)
$$

such that

$$
\varrho(g) \leqslant i(\mathscr{Q})+\varepsilon \quad \text { and } \quad \int_{I} \log |g(t)| d t \geqslant 0 .
$$

Of course, we may assume that $c_{0} \neq 0$. Further, we can find a trigonometric polynomial

$$
h(t)=\sum_{k=0}^{n} a_{k} e^{2 \pi i k t}
$$

such that $|h(t)|=|g(t)|$ for $t \epsilon I$ and the polynomial $\sum_{k=0}^{n} a_{k} z^{k}$ has no zero inside the unit circle. By the Jensen equation and (1.7) we have

$$
\log \left|a_{0}\right|=\int_{I} \log |h(t)| d t \geqslant 0 .
$$

Hence and from (1.7) we obtain the inequality $\varrho\left(a_{0}^{-1} h\right) \leqslant i(\mathscr{Q})+\varepsilon$. Since $a_{0}^{-1} h \epsilon \mathscr{P}$, we infer that for every positive number $\varepsilon$ the inequality $i(\mathscr{P}) \leqslant i(\mathscr{Q})+\varepsilon$ holds. Thus $i(\mathscr{P}) \leqslant i(\mathscr{Q})$, which completes the proof.

LEMMA 1.2. If the Lebesgue measure is not absolutely continuous with respect to the measure $v$, then $i(\mathscr{P})=0$.

Proof. Let $E$ be a Borel subset of $I$ such that $|E|>0$ and $v(E)=0$, where $|E|$ denotes the Lebesgue measure of $E$. Given $\varepsilon>0$, we can find a positive number $c$ such that $\varrho(c)>\varepsilon$. Taking a positive number $q$ satisfying the condition $\log q \geqslant|E|^{-1}|\log c|$, we put $g=c+q h_{I}$, where $h_{L}$ is the indicator of the set $E$. Evidently, $\varrho(g)=\varrho(c)<\varepsilon$ and $\int_{I} \log g(t) d t \geqslant 0$. Thus $i(\mathscr{Q})=0$ and, consequently, by Lemma 1.1, $i(\mathscr{P})=0$ which completes the proof.

LEMMA 1.3. If the Lebesgue measure is absolutely continuous with respect to the measure $v$ and $\log \Omega_{\phi, v, p}$ is Lebesgue integrable over $I$ for an index $p$, then $i(\mathscr{P})>0$.
Proof. Contrary to this let us assume that $i(\mathscr{P})=0$. Put

$$
a=\int_{I} \log \Omega_{\Phi, p, p}(t) d t .
$$

Let $k$ be an integer satisfying the inequality

$$
k>\frac{a+1}{\log 2} .
$$

Further, let $g$ be a trigonometric polynomial from $\mathscr{P}$ satisfying the inequalities

$$
\varrho(g)<\frac{1}{p x^{k}}, \quad \int_{I} \log |g(t)| d t \geqslant 0,
$$

where $x$ is the constant appearing in the $\Delta_{2}$-condition for the function $\Phi$. Setting $h=2^{k} g$ and $E=\left\{t:|h(t)|>\Omega_{\Phi, v, p}(t)\right\}$, we have, by virtue of (1.2), (1.3) and (1.4), the inequality

$$
\log |h(t)| \frac{d t}{d \nu} \leqslant p \Phi(t,|h(t)|) \quad(t \in E) .
$$

Thus

$$
\int_{E} \log |h(t)| d t \leqslant p \int_{E} \Phi(t,|h(t)|) v(d t) \leqslant p \varrho(h) .
$$

On the other hand, by the $\Delta_{2}$-condition and (1.9),

and, consequently,

$$
\varrho(h) \leqslant x^{k} \varrho(g)<\frac{1}{p},
$$

$$
\int_{E} \log |h(t)| d t<1 .
$$

Since

$$
\int_{\Lambda \backslash E} \log |h(t)| d t \leqslant \int_{\Lambda \backslash E} \log \Omega_{\Phi, v, p}(t) d t \leqslant a,
$$

we have

$$
\int_{I} \log |h(t)| d t \leqslant a+1
$$

But, by (1.9),

$$
\int_{I} \log |h(t)| d t \geqslant k \log 2
$$

and, consequently, $k \leqslant(a+1) / \log 2$ which contradicts inequality (1.8).

The Lemma is thus proved.

Studia Mathematica XXXI,1 
LEMMA 1.4. If the Lebesgue measure is absolutely continuous with respect to the measure $v$ and no function $\log \Omega_{\Phi, v, n}(n=1,2, \ldots)$ is Lebesgue integrable over $I$, then $i(\mathscr{P})=0$.

Proof. Put

$$
A_{n}=\left\{t: 1<\Omega_{\Phi, v, n}(t)<\infty\right\} \quad(n=1,2, \ldots) .
$$

First consider the case

$$
\int_{A_{1}} \log \Omega_{\Phi, n, n}(t) d t=\infty \quad(n=1,2, \ldots) .
$$

Given $\varepsilon>0$, we take a positive number $c$ for which

$$
\varrho(c) \leqslant \frac{\varepsilon}{2} .
$$

From (1.10) it follows that there exists a subset $B_{n}$ of the set $A_{n}$ for which

$$
\int_{B_{n}} \log \Omega_{\Phi, v, n}(t) d t=\frac{n \varepsilon}{2} \quad(n=1,2, \ldots) .
$$

Since, by (1.2), (1.3) and (1.4),

$$
\log \Omega_{\Phi, v, n}(t) \frac{d t}{d v}=n \Phi\left(t, \Omega_{\phi, v, n}(t)\right) \quad\left(t \in A_{n}\right),
$$

we have, by (1.12), the formula

$$
\int_{B_{n}} \Phi\left(t, \Omega_{\Phi, v, n}(t)\right) v(d t)=\begin{aligned}
& \varepsilon \\
& 2
\end{aligned} \quad(n=1,2, \ldots) .
$$

Put $g_{n}(t)=\Omega_{\Phi, v, n}(t)$ on $B_{n}$ and $g_{n}(t)=c$ otherwise. By (1.11) and (1.13), we have the inequality $\varrho\left(g_{n}\right) \leqslant \varepsilon$. Moreover, by (1.12),

$$
\int_{I} \log g_{n}(t) d t=\frac{n \varepsilon}{2}+\left|I \backslash B_{n}\right| \log c .
$$

Thus $g_{n} \epsilon \mathscr{Q}$ for $n \geqslant 2|\log c| / \varepsilon$ and, consequently, $i(\mathscr{2}) \leqslant \varepsilon$ whence the formula $i(\mathscr{Q})=0$ follows. Now the assertion of the Lemma is a consequence of Lemma 1.1 .

In the remaining case there exists an index $p$ for which $\log \Omega_{\Phi, v, p}$ is Lebesgue integrable over the set $A_{p}$. Since the sequence $\Omega_{\phi, n, n}$ $(n=1,2, \ldots)$ is monotone non-increasing, the function $\log \Omega_{\phi, n, n}$ is Lebesgue integrable over the set $A_{n}$ for $n \geqslant p$. Consequently, the sets

$$
C_{n}=\left\{t: \Omega_{\Phi, v, n}(t)=\infty\right\}
$$

have for $n \geqslant p$ positive Lebesgue measure. Given a positive number $\varepsilon$, we take an integer $m$ satisfying the inequalities

$$
m \geqslant p \quad \text { and } \quad m \geqslant \frac{2|\log c|}{\varepsilon},
$$

where the number $c$ is determined by (1.11). Put

$$
q=\exp \left(\frac{\varepsilon}{2} m\left|C_{m}\right|^{-1}\right) \text {. }
$$

By the definitions (1.3), (1.4) and (1.14) the function

$$
h(t)=\inf \left\{x: \frac{\log x}{\Phi(t, x)} \frac{d t}{d v} \geqslant m, x \geqslant q\right\}
$$

is finite on $C_{m}$ and, of course, Borel measurable. Moreover,

$$
\int_{C_{m}} \log h(t) d t \geqslant\left|C_{m}\right| \log q=\frac{m \varepsilon}{2} .
$$

Consequently, there exists a Borel subset $D$ of $C_{m}$ for which

Thus

$$
\int_{D} \log h(t) d t=\frac{m \varepsilon}{2} .
$$

$$
\int_{D} \Phi(t, h(t)) v(d t) \leqslant \frac{1}{m} \int_{D} \log h(t) d t=\frac{\varepsilon}{2} .
$$

Put $g(t)=h(t)$ on $D$ and $g(t)=c$ otherwise. By (1.11) and (1.17) we have the inequality $\varrho(g) \leqslant \varepsilon$. Moreover, by (1.15) and (1.16),

$$
\int_{I} \log g(t) d t=\frac{m \varepsilon}{2}+|I \backslash D| \log c \geqslant 0 .
$$

Thus $i(\mathscr{Q}) \leqslant \varepsilon$ and, consequently, $i(\mathscr{Q})=0$. Taking into account Lemma 1.1, we get the formula $i(\mathscr{P})=0$ which completes the proof.

Proof of the Theorem 1.1. Since the modular convergence and the norm convergence in $L_{\Phi}(v)$ are equivalent, equation (1.5) is equivalent with the equation $i(\mathscr{P})=0$. Consequently, by Lemmas 1.3 and 1.4, the Theorem is true if the Lebesgue measure is absolutely continuous with respect to the measure $\nu$. If the Lebesgue measure is not absolutely continuous with respect to the measure $v$, then $d v_{c} / d t=0$ on a set of positive Lebesgue measure and, consequently, by (1.3) and (1.4), no function $\log \Omega_{\Phi, v, n}(n=1,2, \ldots)$ is Lebesgue integrable over $I$. In this case the Theorem is a consequence of Lemma 1.2, which completes the proof. 
We conclude this section with some particular cases of the Theorem 1.1. If the function $\Phi$ does not depend upon the variable $t$, i.e. $\Phi(t, x)=\Phi(x)$ $\left(t \in I, x \in R_{+}\right), L_{\Phi}(v)$ is called an Orlicz space (see [11] and [13]). In this paper we have assumed the $\Delta_{2}$-condition for $\Phi$. We say that the function $\Phi$ satisfies the $\Lambda_{a}$-condition for a number $a>1$ if there exists a constant $\gamma_{a}>1$ such that

$$
\Phi(x) \gamma_{a} \leqslant \Phi(a x)
$$

for sufficiently large $x$ (see [12]).

Now we shall prove the original version of the Kolmogorov-Krein criterion.

THEOREM 1.2. Let $L_{\Phi}(\nu)$ be an Orlicz space and let $\Phi$ satisfy the $\Lambda_{a}$ condition for some constant $a>1$. Then equation (1.5) holds if and only if $\log \left(d v_{c} / d t\right)$ is not Lebesgue integrable over $I$.

Pro of. Of course, it suffices to consider the case when the Lebesgue measure is absolutely continuous with respect to the measure $v$. Since, by (iv), $v(I)$ is finite, we have the inequality

$$
\int_{I} \log \frac{d v_{c}}{d t} d t<\infty
$$

Thus to prove our statement it suffices to prove that (1.5) is equivalent to the equation

$$
\int_{I} \log \frac{d v_{c}}{d t} d t=-\infty .
$$

From the $\Delta_{2}$-condition and the $\Lambda_{a}$-condition for $\Phi$ it follows that there are positive constants $c_{1}, c_{2}, p$ and $q$ such that

$$
c_{1} x^{2 p} \leqslant \Phi(x) \leqslant c_{2} x^{q}
$$

for sufficiently large $x$ (see [12]). Consequently, we can find a positive number $x_{0}$ such that

$$
c_{1} x^{p} \leqslant \frac{\Phi(x)}{\log x} \leqslant c_{2} x^{\alpha} \quad \text { if } x \geqslant x_{0} .
$$

Hence, in particular, it follows that

$$
\lim _{x \rightarrow \infty} \Lambda_{\Phi, n}(t, x)=0
$$

for almost all $t$ in the sense of the Lebesgue measure, because $d v_{c} / d t$ is almost everywhere positive. Consequently, the functions $\Omega_{p, p, n}$ $(n=1,2, \ldots)$ are finite almost everywhere. Put

$$
F_{n}=\left\{t: \max \left(1, x_{0}\right)<\Omega_{\Phi, v, n}(t)<\infty\right\} \text {. }
$$

Then, by the definitions (1.3) and (1.4), the function $\left(d v_{c} / d t\right)^{-1}$ is bounded on $I \backslash F_{n}$ almost everywhere and

$$
\log \Omega_{\Phi, v, n}(t)\left(\frac{d v_{c}}{d t}\right)^{-1}=n \Phi\left(\Omega_{\Phi, v, n}(t)\right)
$$

for $t \epsilon F_{n}$. Hence and from (1.18) we get the inequalities

$$
n c_{1} \Omega_{\Phi, v, n}^{p}(t) \leqslant\left(\frac{d v_{c}}{d t}\right)^{-1} \leqslant n c_{2} \Omega_{\Phi, v, n}^{q}(t) \quad\left(t \in F_{n}\right) .
$$

Consequently, no function $\log \Omega_{\Phi, p, n}(n=1,2, \ldots)$ is Lebesgue integrable over $I$ if and only if

$$
\int_{I} \log \frac{d v_{c}}{d t} d t=-\infty
$$

Our theorem is now a consequence of Theorem 1.1.

THEOREM 1.3. If $L_{\Phi}(v)$ is an Orlicz space and

$$
\lim _{x \rightarrow \infty} \frac{\Phi(x)}{\log x}=c>0
$$

then equation (1.5) holds if and only if

$$
\text { ess inf }\left\{\frac{d v_{c}}{d t}: t \in I\right\}=0
$$

Proof. If ess inf $\left\{d v_{c} \mid d t: t \in I\right\}=0$, then the set

$$
G_{n}=\left\{t: \frac{d v_{c}}{d t}<\frac{c}{n}\right\}
$$

has for every $n$ a positive Lebesgue measure and, by (1.3) and (1.4), $\Omega_{\Phi, v, n}(t)=\infty$ on $G_{n}$. Hence we infer that no function $\log \Omega_{\Phi, v, n}$ $(n=1,2, \ldots)$ is Lebesgue integrable over $I$.

Suppose now that

$$
\text { ess inf }\left\{\frac{d v_{c}}{d t}: t \epsilon I\right\}=a>0
$$

and put

$$
b=\sup \left\{\frac{\log x}{\Phi(x)}: x \geqslant 1\right\} .
$$

Of course, $b<\infty$ and, by (1.3) and (1.4), $\Omega_{\Phi, v, m}(t)=1$ almost everywhere for all indices $m$ satisfying the inequality $m>a^{-1} b$. Consequently, our theorem is a simple consequence of Theorem 1.1. 
In the same way we can prove the following theorems:

Theorem 1.4. If $L_{\Phi}(v)$ is an Orlicz space and

$$
\lim _{x \rightarrow \infty} \frac{\Phi(x)}{\log ^{p+1} x}>0,
$$

where $p$ is a positive number, then equation (1.5) holds if and only if

$$
\int_{I}\left(\frac{d v_{c}}{d t}\right)^{-1 / p} d t=\infty
$$

THEOREM 1.5. If $L_{\Phi}(p)$ is an Orlice space and

$$
\lim _{x \rightarrow \infty} \frac{\Phi(x)}{\log x \log \log x}>0,
$$

then equation (1.5) holds if and only if

$$
\int_{I} \exp \left\{n^{-1}\left(\frac{d v_{c}}{d t}\right)^{-1}\right\} d t=\infty
$$

for all positive integers $n$.

2. Vector-valued random measures. In this section by $(x, y)$ and $|x|$ we shall denote the inner product and the norm respectively in $R^{p}$. Further, for any $R^{p}$-valued random variable $X, p_{X}(t)\left(t \in R^{p}\right)$ will denote the characteristic function of $X$, i.e. the expectation $\mathrm{E} e^{i(t, X)}$.

A function $M$ defined on the $\sigma$-algebra of all Borel subsets of the unit interval $I$ whose values are $R^{p}$-valued random variables is called an $R^{p}$-valued random measure or shortly a random measure if

(*) for every sequence $E_{1}, E_{2}, \ldots$ of disjoint Borel sets

$$
M\left(\bigcup_{n=1}^{\infty} E_{n}\right)=\sum_{n=1}^{\infty} M\left(E_{n}\right),
$$

where the series converges with probability 1 ,

(**) for every sequence $E_{1}, E_{2}, \ldots, E_{n}$ of disjont Borel sets the random variables $M\left(E_{1}\right), M\left(E_{2}\right), \ldots, M\left(E_{n}\right)$ are independent.

The theory of random measures was developed. by A. Pxéliopa in [15], [16] and [17]. For further results see [6], [22] and [24].

A random measure is said to be atomless if $M(\{a\})=0$ with probability 1 for every one-point set $\{a\}$. In this paper we shall consider atomless random measures only. Moreover, we shall identify random variables which are equal with probability 1 . Given a random measure $M$, we say that a Borel set $E$ is an $M$-null set if $M(A)=0$ for all Borel subsets $A$ of $E$. Relations valid except on an $M$-null set are said to be valid $M$-almost everywhere.
The concept of the integral of a real-valued function with respect to a real-valued random measure was introduced in [16] (the unconditional integral) and in [22]. In an analogous way the integral of an operatorvalued function with respect to a vector-valued random measure was introduced in [24]. We shall quote the basic definition, which is an adaptation of the Dunford's definition of the integral with respect to a measure whose values belong to a Banach space ([4], Chapter IV).

Let $M$ be an atomless $R^{p}$-valued random measure. If $F$ is an operatorvalued Borel simple function on $I$,

$$
F=\sum_{j=1}^{n} C_{j} \chi_{E_{j}},
$$

where $E_{j}$ are Borel sets, $C_{j}$ are linear operators on $R^{p}$ and $\chi_{E_{j}}$ denotes the indicator of $E_{j}(j=1,2, \ldots, n)$, then the integral on every Borel set $E$ of $F$ with respect to $M$ is defined by the formula

$$
\int_{E} F(s) M(d s)=\sum_{j=1}^{n} C_{j} M\left(E_{j} \cap E\right) .
$$

Further, an operator-valued Borel function defined on $I$ is said to be $M$-integrable if there exists a sequence of operator-valued Borel simple functions $\left\{F_{n}\right\}$ such that

$1^{\circ}$ the sequence $\left\{F_{n}\right\}$ converges to $F^{\prime} M$-almost everywhere on $I$,

$2^{\circ}$ for every Borel set $E$ the sequence $\left\{\int_{E} F_{n}(s) M(d s)\right\}$ converges in probability.

Then, by the definition, the integral $\int_{E} F(s) M(d s)$ is the limit in probability of the sequence $\left\{\int_{E} F_{n}(s) M(d s)\right\}$.

A random measure $M$ is said to be symmetric if for every Borel set $E$ the random variables $M(E)$ and $-M(E)$ are identically distributed. Since the values $M(E)$ of an atomless random measure have an infinitely divisible distribution, we infer that for symmetric atomless random measures the characteristic function of the random variable $M(E)$ can be written in the Lévy-Khinchine form

$$
\begin{aligned}
p_{M(E)}(t) & =\exp \left\{-\left(D_{M}(E) t, t\right)-\right. \\
& \left.-\int_{R^{p} \backslash\{0\}}(1-\cos (t, x)) \frac{1+|x|^{2}}{|x|^{2}} \lambda_{M}(E, d x)\right\},
\end{aligned}
$$

where $D_{M}(E)$ is a symmetric non-negative operator on $R^{p}$ and $\lambda_{M}(E, \cdot)$ is a finite non-negative measure on $R^{p} \backslash\{0\}$. Moreover, $D_{M}(\cdot)$ is an operatorvalued Borel measure on $I$ and for every Borel subset $A$ of $R^{p} \backslash\{0\}$ 
the set-function $\lambda_{M}(\cdot, A)$ is a non-negative Borel measure on $I$. In the sequel we shall use the notation

$$
\psi_{X}(t)=-\log \varphi_{X}(t)
$$

for symmetric random variables $X$ with an infinitely divisible distribution. Given a symmetric atomless random measure $M$, we put

(2.3) $\quad Q_{M}(u, v, E)=2 \psi_{M(E)}(u)+2 \psi_{M(E)}(v)-\psi_{M\left(E^{\prime}\right)}(u+v)-\psi_{M(E)}(u-v)$.

By (2.1) we have

$$
Q_{M}(u, v, E)=2 \int_{R^{p} \backslash\{0\}}(1-\cos (u, x))(1-\cos (v, x)) \frac{1+|x|^{2}}{|x|^{2}} \lambda_{M}(E, d x) .
$$

Consequently, $Q_{M}(\cdot, \cdot, E)$ is a continuous function and $Q_{M}(u, v, \cdot)$ is a non-negative Borel measure on $I$. Let us fix an orthonormal basis $e_{1}, e_{2}, \ldots, e_{p}$ in $R^{p}$. Given

$$
x=\sum_{j=1}^{p} \alpha_{j} e_{j} \quad \text { and } \quad y=\sum_{j=1}^{p} \beta_{j} e_{j}
$$

we put

$$
x \circ y=\sum_{j=1}^{p} \alpha_{j} \beta_{j} e_{j}
$$

Further, for any pair $F, G$ of $M$-integrable operator-valued functions we put

(2.4) $S(F, G, u, v)=2 \psi_{u_{\circ} X}(w)+2 \psi_{v_{\circ} Y}(w)-\psi_{u_{\circ} X+v_{\circ} Y}(w)-\psi_{u \circ X-v \circ X}(w)$, where

$$
\begin{gathered}
w=\sum_{j=1}^{p} e_{j}, \quad X=\int_{I} F(s) M(d s), \\
Y=\int_{I} G(s) M(d s) \quad \text { and } \quad u, v \in R^{p} .
\end{gathered}
$$

LemMa 2.1. Let $M$ be a symmetric atomless random measure and let $F, G$ be a pair of operator-valued $M$-integrable functions on $I$. Then for every triplet $a, b, r(a<b)$ of positive numbers we have the inequality

$$
\int_{K_{r}} \int_{K_{r}} S(F, G, u, v) d u d v \geqslant b^{-2 p} \int_{K_{a r}} \int_{K_{a r}} Q_{M}\left(u, v, U_{a, b}(F) \cap U_{a, b}(G)\right) d u d v,
$$

where $K_{c}=\left\{x: x \in R^{p},|x| \leqslant c\right\}, U_{a, b}(H)=\left\{s: K_{a} \subset H^{*}(s) K_{1} \subset K_{b}\right\}, H^{*}(s)$ being the conjugate of $H(s)$.

Proof. By the definition of $M$-integrable functions there are two sequences of operator-valued Borel simple functions $\left\{F_{n}\right\}$ and $\left\{G_{n}\right\}$ which converge to $F$ and $G M$-almost everywhere respectively and.

$$
\lim _{n \rightarrow \infty} \int_{I} F_{n}(s) M(d s)=\int_{I} \boldsymbol{F}(s) M(d s), \quad \lim _{n \rightarrow \infty} \int_{I} G_{n}(s) M(d s)=\int_{I} G(s) M(d s) .
$$

Taking into account definitions (2.2) and (2.4), we infer that

$$
\lim _{n \rightarrow \infty} S\left(F_{n}, G_{n}, u, v\right)=S(F, G, u, v)
$$

uniformly on every compact subset of $R^{p} \times R^{p}$. Consequently,

$$
\lim _{n \rightarrow \infty} \int_{K_{r}} \int_{K_{r}} S\left(F_{n}, G_{n}, u, v\right) d u d v=\int_{K_{r}} \int_{K_{r}} S(F, G, u, v) d u d v .
$$

Moreover, there exists an $M$-null set $U$ such that for every positive number $\varepsilon$ less than $a$ we have the inclusion

(2.6) $\quad \liminf _{n \rightarrow \infty} U_{a-\varepsilon, b+\varepsilon}\left(F_{n}\right) \frown U_{a-\varepsilon, b+\varepsilon}\left(G_{n}\right) \supset U_{a, b}\left(F^{\prime}\right) \frown U_{a, b}(G) \backslash U$.

Let $n$ be fixed for a moment. Introducing the notation

$$
F_{n}=\sum_{j=1}^{k} A_{j} \chi_{E_{j}}, \quad G_{n}=\sum_{j=1}^{k} B_{j} \chi_{E_{j}}
$$

where the Borel sets $E_{1}, E_{2}, \ldots, E_{k}$ are disjoint, we have, by virtue of (2.3) and (2.4), the formula

$$
S\left(F_{n}, G_{n}, u, v\right)=\sum_{j=1}^{k} Q_{M}\left(A_{j}^{*} u, B_{j}^{*} v, E_{j}\right)
$$

Without loss of generality we may assume that

$$
\bigcup_{j=1}^{s} E_{j}=U_{a-\varepsilon, b+\varepsilon}\left(F_{n}\right) \frown U_{a-\varepsilon, b+\varepsilon}\left(G_{n}\right)
$$

where $s \leqslant k$ and $\varepsilon$ is a positive number less than a. Consequently, for $j \leqslant s$ the operators $A_{j}^{*}$ and $B_{j}^{*}$ are invertible, $\left(A_{j}^{*}\right)^{-1} K_{r} \supset K_{(a-\varepsilon) r}$, $\left(B_{j}^{*}\right)^{-1} K_{r} \supset K_{(a-\varepsilon) r}$ and the inequalities $\left|\operatorname{det}\left(A_{j}^{*}\right)^{-1}\right| \geqslant(b+\varepsilon)^{-p},\left|\operatorname{det}\left(B_{j}^{*}\right)^{-1}\right| \geqslant$ $\geqslant(b+\varepsilon)^{-p}$ hold, where the matrix representation of operators with respect to the orthonormal basis $e_{1}, e_{2}, \ldots, e_{p}$ is taken. Hence we get the inequality

$$
\int_{\bar{K}_{r}} \int_{\mathcal{K}_{r}} Q_{M}\left(A_{j}^{*} u, B_{j}^{*} v, E_{j}\right) d u d v \geqslant(b+\varepsilon)^{-2 p} \int_{K_{(a-\varepsilon) r} K_{(a-\varepsilon) r}} \int_{M} Q_{M}\left(u, v, E_{j}\right) d u d v
$$

for $j \leqslant s$. Thus, by (2.7),

$$
\int_{K_{r}} \int_{K_{r}} S\left(F_{n}, G_{n}, u, v\right) d u d v \geqslant(b+\varepsilon)^{-2 p} \int_{K_{(a-\varepsilon) r} K_{(a-\varepsilon) r}} \int_{M} Q_{M}\left(u, v, \bigcup_{j=1}^{s} E_{j}\right) d u d v .
$$

Hence, by (2.5), (2.6), (2.8) and Fatou's Lemma, we get the inequality

$$
\begin{aligned}
\int_{K_{r}} \int_{K_{r}} S(F, Q, u, v) d u d v & \\
& \geqslant(b+\varepsilon)^{-2 p} \int_{K_{(a-8) r} K_{(a-s) r}} \int_{M} Q_{M}\left(u, v, U_{a, b}(F) \frown U_{a, b}(G)\right) d u d v .
\end{aligned}
$$


Since $\varepsilon$ is an arbitrary positive number, from the last inequality we obtain the assertion of the Lemma.

Now we shall prove a continuous analogue of the Bernstein-Darmois theorem ([1], [2]). For homogeneous random measures this problem was discussed in [9], [18] and [21].

THEOREM 2.1. Let $M$ be a vector-valued atomless random measure and let $F$ and $G$ be operator-valued $M$-integrable functions on $I$. If the integrals $\int F(s) M(d s)$ and $\int_{I} G(s) M(d s)$ are independent, then for any Borel subset $E$ of the set $\left\{s: F^{\prime}(s)\right.$ and $G(s)$ are invertible $\}$ the random variables $M(E)$ are Gaussian.

Proof. By Cramér's Theorem ([10], p. 271) we may assume, without loss of generality, that the random measure $M$ is symmetric. Moreover, it suffices to prove that the random variable $M(U)$ is Gaussian, where

$$
U=\{s: F(s) \text { and } G(s) \text { are invertible }\} .
$$

Since the integrals $\int_{I} F(s) M(d s), \int_{I} G(s) M(d s)$ are independent and symmetrically distributed, we have, by definition (2.4), the equation $S(F, G, u, v)=0$ for all $u, v \in R^{p}$. Consequently, by Lemma 2.1,

$$
\int_{K_{a r}} \int_{K_{a r}} Q_{M r}\left(u, v, U_{a, b}\left(F^{\prime}\right) \frown U_{a, b}(G)\right) d u d v=0
$$

for all positive numbers $a, b$ and $r(a<b)$. Taking into account the continuity of $Q_{M}(\cdot, \cdot, E)$ we infer that

$$
Q_{M}\left(u, v, U_{a, b}(F) \frown U_{a, b}(G)\right)=0
$$

for all $u, v \in R^{p}$ and $b>a>0$. Since $U=\bigcup U_{a, b}(F) \cap U_{a, b}(G)$, where the union is taken over all pairs $a<b$ of positive rational numbers, we finally get the equation

$$
Q_{M}(u, v, U)=0 \quad\left(u, v \in R^{p}\right) .
$$

Consequently, by $(2.3)$, the function $f(t)=\psi_{M(U)}(t)$ is a non-negative continuous solution of the functional equation

$$
2 f(u)+2 f(v)-f(u+v)-f(u-v)=0 \quad\left(u, v \in R^{p}\right) .
$$

It is well-known (see [5]) that each non-negative continuous solution of this equation is of the form $f(t)=(D t, t)$, where $D$ is a non-negative symmetric operator. Hence it follows that the random variable is Gaussian which completes the proof.

3. Complex-valued isotropic random measures. In this section we identify the complex plane and the space $R^{2}$. The integral of a complex- valued function $f$ with respect to a complex-valued random measure $M$ is defined by means of the formula

$$
\int_{I} f(s) M(d s)=\int_{I} \hat{f}(s) M(d s),
$$

where $\hat{z}$ is a matrix representation of the complex number $z$ given by the formula

$$
\hat{z}=\left(\begin{array}{rr}
\operatorname{Re} z & -\operatorname{Im} z \\
\operatorname{Im} z & \operatorname{Re} z
\end{array}\right) .
$$

A complex-valued random measure $M$ is said to be isotropic if for every orthogonal operator $V$ in $R^{2}$ and every Borel subset $E$ of $I$ the random variables $M(E)$ and $V M(E)$ have the same probability distribution. For an atomless isotropic random measure $M$ the characteristic function of the random variable $M(E)$, where $E$ is a Borel subset of $I$, can be written in the form

$$
\varphi_{M(E)}(t)=\exp \left\{\int_{0}^{\infty}\left(J_{0}(x|t|)-1\right) \frac{1+x^{2}}{x^{2}} \mu_{M I}(E, d x)\right\},
$$

where $J_{0}$ is the Bessel function defined by

$$
J_{0}(x)=\frac{1}{\pi} \int_{0}^{\pi} \cos (x \sin u) d u
$$

and $\mu_{M}(E, \cdot)$ is a finite non-negative Borel measure on $R_{+}$(see [5]). Moreover, for every Borel subset $A$ of $R_{+}$the set-function $\mu_{M}(\cdot, A)$ is a non-negative Borel measure on $I$. Put $v_{M}(E)=\mu_{M}\left(E, R_{+}\right)$. It is obvious that $\mu_{M}(\cdot, A) \leqslant \nu_{M}$ and, consequently, all measures $\mu_{M}(\cdot, A)$ are absolutely continuous with respect to the measure $v_{M}$. Put $G_{M}(E, x)$ $=\mu_{M}(E,[0, x)), x \in R_{+}$. By the Radon-Nikodym theorem

$$
G_{M}(E, x)=\int_{E} g_{M}(s, x) v_{M}(d s),
$$

where $0 \leqslant g_{M}(s, x) \leqslant 1$ and the function $g_{M}(\cdot, x)$ is Borel measurable. Moreover, we may assume that the function $g_{M}(s, \cdot)$ is monotone nondecreasing and continuous to the left on $R_{+}$. In fact, we can always find a version $\tilde{g}_{M}(\cdot, w)$ of the Radon-Nikodym densities of $G_{M}(\cdot, w)$ for rational numbers $w$, such that $\tilde{g}_{M}(\cdot, w)$ is Borel measurable and monotone non-decreasing as the function of $w$. Setting

$$
g_{M}(s, x)=\lim _{w \rightarrow x_{-}} \tilde{g}_{M}(s, w)
$$

we obtain a Radon-Nikodym density with required properties. 
Put

$$
\Phi_{M}(t, x)=\int_{1 / x}^{\infty} \frac{g_{M}(t, u)}{u^{3}} d u \quad\left(t \in I, x \in R_{+}\right) .
$$

The function $\Phi_{M}$ and the measure $v_{M}$ corresponding to the random measure $M$ satisfy conditions (i)-( $v$ ) from section 1 . The $\Delta_{2}$-condition for $\Phi_{M}$ is a consequence of the inequality

$$
\begin{aligned}
\Phi_{M}(t, 2 x) & =\int_{1 / 2 x}^{\infty} \frac{g_{M}(t, u)}{u^{3}} d u=4 \int_{1 / x}^{\infty} \frac{g_{M}\left(t, \frac{1}{2} u\right)}{u^{3}} d u \\
& \leqslant 4 \int_{1 / x}^{\infty} \frac{g_{M}(t, u)}{u^{3}} d u=4 \Phi_{M}(t, x) .
\end{aligned}
$$

Let $\mathscr{L}(M)$ be the set of all complex-valued $M$-integrable functions, where $M$ is a complex-valued atomless isotropic random measure. We identify functions which are equal $M$-almost everywhere. The space $\mathscr{L}(M)$ is a complete linear metric space under usual addition and scalar multiplication with a non-homogeneous norm defined by the formula

$$
\|f\|_{M}=\left\|\int_{I} f(s) M(d s)\right\|
$$

where $\||X|\|$ denotes the Fréchet norm of the random variable $X$, i.e. the expectation $\mathrm{E}[(1+|X|) /|X|]$ (see [22] and [24]). It should be noted. that the convergence of a sequence of functions in $\mathscr{L}(M)$ is equivalent to the convergence in probability of the sequence of their integrals with respect to the random measure $M$. Moreover, the set of all Borel simple functions on $I$ is dense in $\mathscr{L}(M)$.

By (3.1), $\varphi_{M(E)}(t)$ depends only upon the modulus of $t$. Consequently, we can introduce the notation

$$
\vartheta_{M(E)}(|t|)=-\log \varphi_{M(E)}(t)
$$

LEMMA 3.1. Let $M$ be a complex-valued atomless isotropic random measure. There exists then a positive constant $c_{1}$ such that the inequality

$$
\int_{0}^{1} \vartheta_{M(E)}(a r) d r \geqslant c_{1} \int_{E} \Phi_{M}(s, a) v_{M}(d s)
$$

holds for all non-negative numbers $a$ and all Borel subsets $E$ of I. Moreover, for every positive number $\varepsilon$ there exist a positive constant $c_{2}$ and a Borel subset $A$ of $R_{+}$such that $\mu_{M}(I, A)<\varepsilon$ and the inequality

$$
\vartheta_{M(E)}(a) \leqslant c_{2} \int_{E} \Phi_{M}(s, a) v_{M}(d s)+2 \mu_{M}(E, A)
$$

holds for all non-negative numbers a and all Borel subsets $E$ of $I$.
Proof. Integrating by parts, from (3.3) and (3.4) we get the formula

$$
\int_{E} \Phi_{M}(s, a) \nu_{M}(d s)=\frac{1}{2} \int_{0}^{\infty} \min \left(a^{2}, x^{-2}\right) \mu_{M}(E, d x) .
$$

Taking into account the well-known inequality

$$
1-\frac{\sin y}{y} \geqslant b_{1} \min \left(1, y^{2}\right)
$$

where $b_{1}$ is a positive constant, we get, by (3.2), the inequality

$$
\int_{0}^{1}\left(1-J_{0}(a x r)\right) d r \geqslant b_{2} \min \left(a^{2} x^{2}, 1\right),
$$

where $b_{2}$ is a positive constant. Hence and from (3.1), (3.5) and (3.8) we obtain the inequality

$\int_{0}^{1} \vartheta_{M(E)}(a r) d r \geqslant b_{2} \int_{0}^{\infty} \min \left(a^{2} x^{2}, 1\right) \frac{1+x^{2}}{x^{2}} \mu_{M}(E, d x) \geqslant 2 b_{2} \int_{E} \Phi_{M}(s, a) v_{M}(d s)$.

Formula (3.6) is thus proved.

Given $\varepsilon>0$, we take a number $q \geqslant 1$ such that $\mu_{M}(I, A)<\varepsilon$, where $A=(q, \infty)$. It is clear that

$$
\int_{a}^{\infty}\left(1-J_{0}(a x)\right) \frac{1+x^{2}}{x^{2}} \mu_{M}(E, d x) \leqslant 2 \mu_{M}(E, A)
$$

for all non-negative numbers $a$ and all Borel sets $E$. Further, taking into account the inequality $1-\cos y \leqslant b_{3} \min \left(1, y^{2}\right)$, where $b_{3}$ is a positive constant, we get, by (3.2), the inequality

$$
1-J_{0}(a x) \leqslant b_{3} \min \left(a^{2} x^{2}, 1\right) \text {. }
$$

Consequently, by (3.8), for every non-negative number $a$ and every Borel set $E$ we have the inequality

$$
\begin{aligned}
\int_{q}^{\infty}\left(1-J_{0}(a x)\right) \frac{1+x^{2}}{x^{2}} \mu_{M}(E, d x) & \leqslant b_{3} \int_{0}^{q} \min \left(a^{2}, x^{-2}\right)\left(1+x^{2}\right) \mu_{M}(E, d x) \\
& \leqslant c_{2} \int_{E} \Phi_{M}(s, a) v_{M}(d s),
\end{aligned}
$$

where $c_{2}=2 b_{3}\left(1+q^{2}\right)$. Hence and from (3.1), (3.5) and (3.9) we get inequality (3.7) which completes the proof of the Lemma.

LEMMA 3.2. Let $M$ be a complex-valued atomless isotropic random measure. $A$ sequence $\left\{f_{n}\right\}$ of Borel simple functions on I converges to 0 in $\mathscr{L}(M)$ if and only if it converges to 0 in the Musielak-Orlicz space $L_{\Phi_{M}}\left(v_{M}\right)$. 
Pro of. Given a Borel simple function $f$ on $I$, say

$$
f=\sum_{j=1}^{k} a_{j} \chi_{E_{j}}
$$

where the sets $E_{1}, E_{2}, \ldots, E_{k}$ are disjoint, we put

$$
X_{f}=\int_{I} f(s) \cdot M(d s)=\sum_{j=1}^{k} a_{j} M I\left(E_{j}\right)
$$

Since the characteristic function $\varphi_{X_{f}}(t)$ depends only upon the modulus of $t$, we can introduce the notation $H_{f}(|t|)=-\log \varphi_{X_{j}}(t)$. Further, by (3.5), we have

$$
H_{f}(r)=\sum_{j=1}^{k} \vartheta_{M\left(H_{j}\right)}\left(\left|a_{j}\right| r\right)
$$

By $\varrho_{M}$ we shall denote the modular induced by $\Phi_{M}$ and $v_{M}$ (see definition (1.1)). From (3.10) and Lemma 3.1, for every Borel simple function $f$ we get the inequality

$$
\int_{0}^{1} H_{f}(r) d r \geqslant c_{1} \sum_{j=1}^{k} \int_{E_{j}} \Phi_{M}\left(s,\left|a_{j}\right|\right) v_{M}(d s)=c_{1} \varrho_{M}(f),
$$

where $c_{1}^{\circ}$ is a positive constant. Moreover, for every positive number $\varepsilon$ there exists a positive constant $c_{2}$ such that

$$
H_{f}(r) \leqslant c_{2} \varrho_{M}(f)+2 \varepsilon \text {. }
$$

Suppose that a sequence $\left\{f_{n}\right\}$ of Borel simple functions converges to 0 in $\mathscr{L}(M)$. Then the sequence of random variables $X_{f_{n}}$ converges to 0 in probability. Consequently,

$$
\lim _{n \rightarrow \infty} H_{f_{n}}(r)=0
$$

uniformly in every compact interval. Hence, by (3.11), we get the formula

$$
\lim _{n \rightarrow \infty} \varrho_{M}\left(f_{n}\right)=0
$$

which shows that the sequence $\left\{f_{n}\right\}$ converges to 0 in $L_{\phi_{M}}\left(v_{M}\right)$.

Suppose now that the sequence $\left\{f_{n}\right\}$ of simple functions converges to 0 in $L_{\Phi_{M}}\left(v_{M}\right)$, i.e.

$$
\lim _{n \rightarrow \infty} \varrho_{M}\left(f_{n}\right)=0 .
$$

Since the functions $H_{f_{n}}$ are non-negative, we have, by (3.12), the formula

$$
\lim _{n \rightarrow \infty} H_{f_{n}}\left(r^{n}\right)=0
$$

for every $r$. Consequently, the sequence $\left\{X_{f_{n}}\right\}$ tends to 0 in probability which implies the convergence of $\left\{f_{n}\right\}$ to 0 in $\mathscr{L}(M)$. The Lemma is thus proved.

In this paper two linear metric spaces $\left(Y,\|\|_{1}\right)$ and $\left(Y,\|\|_{2}\right)$ will be treated as identical if the convergence in the norm \|\|$_{1}$ is equivalent to the convergence in the norm \|\|$_{2}$. Since the set of Borel simple functions on $I$ is dense in both spaces $\mathscr{L}(M)$ and $I_{\Phi_{M}}\left(v_{M}\right)$, we have, by Lemma 3.2 , the following characterization of the space $\mathscr{L}(M)$ of all $M$-integrable functions:

THEOREM 3.1. For each complex-valued atomless isotropic random measure $M$ the space $\mathscr{L}(M)$ is identical with the Musielak-Orlicz space $L_{\Phi_{M}}\left(\nu_{M}\right)$.

4. Harmonizable sequences. All random measures considered in this section are assumed to be atomless and complex-valued. The sequence

$$
X_{n}(M)=\int_{0}^{1} e^{2 \pi n i s} M(d s) \quad(n=0, \pm 1, \pm 2, \ldots)
$$

of the Fourier coefficients of a random measure $M$ will be called a harmonizable sequence of random variables. We say that two sequences $\left\{X_{n}\right\}$ and $\left\{\boldsymbol{Y}_{n}\right\}$ of random variables are identically distributed if for every system $n_{1}, n_{2}, \ldots, n_{k}$ of integers the multivariate distributions of $X_{n_{1}}, X_{n_{2}}, \ldots, X_{n_{k}}$ and $Y_{n_{1}}, Y_{n_{2}}, \ldots, Y_{n_{k}}$ are identical. Further, we say that two random measures $M_{1}$ and $M_{2}$ are identically distributed if for every Borel set $\boldsymbol{E}$ the random variables $M_{1}(E)$ and $M_{2}(E)$ are identically distributed. A sequence $\left\{X_{n}\right\}$ of random variables is called strictly stationary, or, shortly, stationary, if for every system $m, n_{1}, n_{2}, \ldots, n_{k}$ of integers the multivariate distribution of the random variables $X_{n_{1}+m}, X_{n_{2}+m}, \ldots, X_{n_{k+m}}$ is independent of $m$.

Let $J$ be an arbitrary subinterval of $I$. Denoting by $\sigma_{n}(\cdot, J)$ the sequence of Fejér means of the Fourier series of the indicator $\chi_{J}(\cdot)$, we inferthat the functions $\sigma_{n}(\cdot, J)$ are bounded in common and

$$
\lim _{n \rightarrow \infty} \sigma_{n}(s, J)=\chi_{J}(s)
$$

except of the endpoints of $J$ (see [25], p. 45). Consequently, by dominated convergence theorem for random integrals ([16], Theorem 2.9), we have

$$
\lim _{n \rightarrow \infty} \int_{I} \sigma_{n}(s, J) M(d s)=M(J) \text { in probability. }
$$

Since the random variables $\int_{I} \sigma_{n}(s, J) M(d s)$ are linear combinations of the Fourier coefficients $X_{k}(M)(|k| \leqslant n)$, we get, by the last formula, the following two Lemmas: 
LEMMA 4.1. The Fourier coefficients $\left\{X_{n}(M)\right\}$ determine the random measure $M$ uniquely.

Lemma 4.2. The random measures $M_{1}{ }^{\circ}$ and $M_{2}$ are identically distributed if and only if the sequences $\left\{X_{n}\left(M_{1}\right)\right\}$ and $\left\{X_{n}\left(M_{2}\right)\right\}$ of their Fourier coefficients are identically distributed.

Now we shall give a characterization of stationary harmonizable sequences of random variables.

THEOREM 4.1. A sequence $\left\{X_{n}(M)\right\}$ is stationary if and only if the random measure $M$ is isotropic.

Proof. Suppose that the sequence $\left\{X_{n}(M)\right\}$ is stationary. Then, by Lemma 4.2 , for every integer $k$ the random measures

$$
M_{k}(E)=\int_{\mathbb{E}} e^{2 \pi k i s} M(d s)
$$

and $M$ are identically distributed. Further, the characteristic function $\varphi_{M(E)}(t)\left(t \in R^{2}\right)$ can be written in the Léry-Khinchine form

$$
\begin{aligned}
\varphi_{M(E)}(t)=\exp \{ & (a(E), t)-(D(E) t, t)+ \\
& \left.+\int_{R^{2} \backslash\{0\}}\left(e^{i(t, x)}-1-\frac{i(t, x)}{1+|x|^{2}}\right) \frac{1+|x|^{2}}{|x|^{2}} \mu(E, d x)\right\},
\end{aligned}
$$

where $a(E)$ is an element of $R^{2}, D(E)$ is a symmetric non-negative operator on $R^{2}$ and $\mu(E, \cdot)$ is a finite non-negative Borel measure on $R^{2} \backslash\{0\}$. More over, $a(\cdot)$ is a vector-valued Borel measure on $I$ and for every Borel subset $A$ of $R^{2} \backslash\{0\}$ the set-function $\mu(\cdot, A)$ is a non-negative Borel measure on $I$. Let us denote by $\mu_{1}(\cdot)$ and $\mu_{2}(\cdot)$ the scalar variations of the measures $a(\cdot)$ and $D(\cdot)$ respectively. Put

$$
\lambda(E)=\mu\left(E, R^{2} \backslash\{0\}\right)+\mu_{1}(E)+\mu_{2}(E) .
$$

Since the random measure $M$ is atomless, we infer that the measure $\lambda$ is also atomless. Moreover, all measures $a(\cdot), D(\cdot)$ and $\mu(\cdot, A)$ are absolutely continuous with respect to the measure $\lambda$. Oonsequently, by the Radon-Nikodym theorem

$$
\begin{gathered}
a(E)=\int_{E} b(s) \lambda(d s), \quad D(E)=\int_{E} O(s) \lambda(d s), \\
\mu(E, A)=\int_{E} h(s, A) \lambda(d s)
\end{gathered}
$$

where $b$ is a vector-valued Borel function, $C$ is an operator-valued. Borel function, $0 \leqslant h(s, A) \leqslant 1$ and the function $h(\cdot, A)$ is Borel measurable. Móreover, we may assume that the set-function $h(s, \cdot)$ is a Borel measure on $R^{2} \backslash\{0\}$. In fact, we can always find a version $g\left(\cdot, w_{1}, w_{2}\right)$ of the Radon-
Nikodym densities of $\mu\left(\cdot, A\left(w_{1}, w_{2}\right)\right)$, where $w_{1}$ and $w_{2}$ are rational numbers and

$$
A\left(w_{1}, w_{2}\right)=\left\{\left(t_{1}, t_{2}\right): t_{1}<w_{1}, t_{2}<w_{2},\left(t_{1}, t_{2}\right) \neq(0,0)\right\},
$$

such that $g\left(\cdot, w_{1}, w_{2}\right)$ is Borel measurable and monotone non-decreasing with respect to each variable $w_{1}$ and $w_{2}$. Furthermore, we may assume that

$$
g\left(\cdot, w_{1}, w_{2}\right)-g\left(\cdot, v_{1}, w_{2}\right)-g\left(\cdot, w_{1}, v_{2}\right)+g\left(\cdot, v_{1}, v_{2}\right) \geqslant 0
$$

whenever $v_{1} \leqslant w_{1}$ and $v_{2} \leqslant w_{2}$. Setting

$$
h\left(s, A\left(u_{1}, u_{2}\right)\right)=\lim _{\substack{w_{1} \rightarrow u_{1}-\\ w_{2} \rightarrow u_{2}-}} g\left(s, w_{1}, w_{2}\right)
$$

for every pair $u_{1}, u_{2}$ of real numbers we get a distribution function which uniquely determines the measure $h(s, \cdot)$.

Put

$$
\begin{aligned}
& K(s, t)=(b(s), t)-(C(s) t, t)+ \\
& \quad+\int_{R^{2} \backslash\{0\}}\left(e^{i(t, x)}-1-\frac{i(t, x)}{1+|x|^{2}}\right) \frac{1+|x|^{2}}{|x|^{2}} h(s, d x) .
\end{aligned}
$$

It is clear that the function $K(\cdot, t)$ is Borel measurable and the function $K(s, \cdot)$ is continuous on $R^{2}$. Moreover, by (4.1) and (4.2),

$$
\varphi_{M(E)}(t)=\exp \int_{E} K(s, t) \lambda(d s) .
$$

Hence it follows that for any Borel simple function $f$ the characteristic function of the integral $\int_{E} f(s) M(d s)$ is given by the expression

$$
\exp \int_{E} K(s, \overline{f(s)} t) \lambda(d s)
$$

where $\bar{z}$ denotes the complex conjugate of $z$. Taking a bounded sequence of Borel simple functions convergent to $e^{2 \pi k i s}$ everywhere on $I$, we infer, by the dominated convergence theorem for random integrals ([16], Theorem 2.9) that the corresponding sequence of integrals over the set $\boldsymbol{E}$ converges in probability to $M_{k}(E)$. Consequently, by the continuity of $K(s, \cdot)$, we have

$$
\varphi_{M_{k}(E)}(t)=\exp \int_{E} K\left(s, e^{-2 \pi k i s} t\right) \lambda(d s) .
$$

Hence and from (4.3) it follows that for every integer $k, t \in R^{2}$ and for $\lambda$-almost all $s$ the equation

$$
K\left(s, e^{-2 \pi k i s} t\right)=K(s, t)
$$


holds. Given $t=|t| e^{2 \pi i \tau}$, we denote by $B$ the subset of $I$ consisting of all irrational numbers $s$ for which equation (4.4) holds for all integers $k$. Since the measure is atomless, we have the formula $\lambda(I \backslash B)=0$. Since for every $s \in B$ the sequence of multiples $s, 2 s, 3 s, \ldots(\bmod 1)$ is dense in $I$ (see for instance [23]), we can take a sequence $\left\{k_{n}\right\}$ of integers for which

$$
\lim _{n \rightarrow \infty} e^{2 \pi k} n_{n}^{i s}=e^{2 \pi i \tau} .
$$

Consequently, by (4.4) and the continuity of $K(s, \cdot)$, we have the formula $K(s, t)=K(s,|t|), s \in B$. Thus, by (4.3), the characteristic function $\varphi_{M_{(E)}(t)}(t)$ depends only upon the modulus of $t$ which implies that the random measure $M$ is isotropic.

Since for isotropic random measures $M$ the characteristic function of the integral $\int_{I} f(s) M(d s)$ depends only upon the absolute value of $f$, the converse implication is obvious. The theorem is thus proved.

Given a stationary sequence $\left\{X_{n}\right\}$, by $\left[X_{n}\right]$ and $\left[X_{n}: n \leqslant k\right]$ we shall denote the linear spaces closed with respect to the convergence in probability spanned by all random variables $X_{n}$ and by random variables $X_{n}$ with $n \leqslant l_{i}$ respectively. To each stationary sequence $\left\{X_{n}\right\}$ there corresponds a shift transformation $T X_{n}=X_{n+1}(n=0, \pm 1, \pm 2, \ldots)$, which can be extended to an invertible linear transformation $T$ on $\left[X_{n}\right]$. Of course, the transformation $T$ preserves the probability distribution.

The concept of stationary sequences admitting a prediction was introduced and discussed in [19]. We say that a stationary sequence $\left\{X_{n}\right\}$ admits a prediction, if there exists a continuous linear operator $A_{0}$ from $\left[X_{n}\right]$ onto $\left[X_{n}: n \leqslant 0\right]$ such that

(i) $A_{0} X=X$ whenever $X \in\left[X_{n}: n \leqslant 0\right]$;

(ii) if for every $Y \in\left[X_{n}: n \leqslant 0\right]$ the random variables $X$ and $Y$ are independent, then $A_{0} X=0$;

(iii) for every $X \in\left[X_{n}\right]$ and $Y \in\left[X_{n}: n \leqslant 0\right]$ the random variables $X-A_{0} X$ and $Y$ are independent.

The random variable $A_{0} X$ can be regarded as a linear prediction. of $X$ based on the full past of the sequence $\left\{X_{n}\right\}$ up to the time $n=0$. An optimality criterion is given by (iii). In what follows the operator $A_{0}$ will be called a predictor based on the past of the sequence $\left\{X_{n}\right\}$ up to the time $n=0$.

It should be noted that Gaussian stationary sequences with zero mean always admit a prediction. This follows from the fact that in this case the concepts of independence and orthogonality are equivalent and, moreover, the square-mean convergence and the convergence in probability are equivalent. Therefore the predictor $A_{0}$ is simply the best linear least squares predictor, i.e. the orthogonal projector from $\left[X_{n}\right]$ onto $\left[X_{n}: n \leqslant 0\right]$ (see [3], Chapter XII, §1).

The predictor $A_{0}$ and the shift $T$ induced by $\left\{X_{n}\right\}$ determine the predictor $A_{k}$ based on the full past of $\left\{X_{n}\right\}$ up to the time $n=k$ by means of the formula $A_{k}=T^{k} A T^{-k}$.

A stationary sequence $\left\{X_{n}\right\}$ admitting a prediction is called deterministic, if $A_{0} X=X$ for every $X \epsilon\left[X_{n}\right]$. Further, a stationary sequence $\left\{X_{n}\right\}$ admitting a prediction is called completely non-deterministic, if $\lim A_{k} X=0$ for every $X \in\left[X_{n}\right]$.

Consider a stationary harmonizable sequence $\left\{X_{n}(M)\right\}$. By Theorem 4.1, the random measure $M$ is isotropic. Further, by Theorem 3.1, $L(M)$ is a Musielak-Orlicz space. It is clear that the mapping $X_{n}(M) \rightarrow e^{2 \pi n i s}$ $(n=0, \pm 1, \pm 2, \ldots)$ can be extended in a natural way to an isomorphism of $\left[X_{n}(M)\right]$ and $\mathscr{L}(M)$. Moreover,

$$
\left[X_{n}(M)\right]=\left\{\int_{I} f(s) M(d s): f \epsilon \mathscr{L}(M)\right\}
$$

and

$$
T \int_{I} f(s) M(d s)=\int_{I} e^{2 \pi i s} f(s) M(d s) .
$$

THEOREM 4.2. Let $\left\{X_{n}(M)\right\}$ be a stationary harmonizable sequence admitting a prediction. There exists then a Borel subset $Q$ of $I$ such that $\left\{X_{n}\left(M_{1}\right)\right\}$ and $\left\{X_{n}\left(M_{2}\right)\right\}$, where $M_{1}(E)=M(E \cap Q)$ and $M_{2}(E)=$ $M(E \cap(I \backslash Q))$, are stationary sequences admitting a prediction. Moreover, the sequence $\left\{X_{n}\left(M_{1}\right)\right\}$ is completely non-deterministic and the sequence $\left\{X_{n}\left(M_{2}\right)\right\}$ is deterministic. Consequently, each stationary harmonizable sequence admitting a prediction is the sum of two independent stationary harmonizable sequences admitting a prediction, one completely non-deterministic and the other deterministic.

Proof. By Theorem 1 in [19], $X_{n}(M)=X_{n}^{\prime}+X_{n}^{\prime \prime}$, where the sequences $\left\{X_{n}^{\prime}\right\}$ and $\left\{X_{n}^{\prime \prime}\right\}$ are independent, stationary and admit a prediction. Moreover, the sequence $\left\{X_{n}^{\prime}\right\}$ is completely non-deterministic, the sequence $\left\{X_{n}^{\prime \prime}\right\}$ is deterministic and the space $\left[X_{n}(M)\right]$ is a direct sum of the subspaces $\left[X_{n}^{\prime}\right]$ and $\left[X_{n}^{\prime \prime}\right]$. Further, $T X_{n}^{\prime}=X_{n+1}^{\prime \prime}$ and $T X_{n}^{\prime \prime}=X_{n+1}^{\prime \prime}$, where $T$ is the shift transformation induced by $\left\{X_{n}(M)\right\}$ in $\left[X_{n}(M)\right]$. Put $X_{0}^{\prime}=\int_{I} h(s) M(d s)$. Consequently,

$$
T^{n} X_{0}^{\prime}=X_{n}^{\prime}=\int_{I} e^{2 \pi n i s} h(s) M(d s) \quad(n=0, \pm 1, \pm 2, \ldots) .
$$

Let us introduce the notation $Q=\{s: h(s) \neq 0\}$. It is evident that

$$
\left[X_{n}^{\prime}\right] \subset\left\{\int_{Q} f(s) M(d s): f \in \mathscr{L}(M)\right\} .
$$


Since for every trigonometric polynomial $w$ the relation

$$
\int_{Q} w(s) h(s)(s) M(d s) \epsilon\left[X_{n}^{\prime}\right]
$$

holds and, by dominated convergence theorem for random integrals (see [16], Theorem 2,9), for every $f \epsilon \mathscr{L}(I M)$ there exists a sequence $\left\{w_{n}\right\}$ of trigonometric polynomials such that

$$
\lim _{n \rightarrow \infty} \int_{Q} w_{n}(s) h(s) M(d s)=\int_{Q} f(s) M(d s)
$$

in probability, we have, by (4.5), the formula

$$
\left[X_{n}^{\prime}\right]=\left\{\int_{Q} f(s) M(d s): f \in \mathscr{L}(M)\right\} .
$$

Since $X_{0}^{\prime \prime}=X_{0}(M)-X_{0}^{\prime}$, we have

$$
X_{0}^{\prime \prime}=M(I \backslash Q)+\int_{Q}(1-h(s)) M(d s) .
$$

Obviously, the random variables $M(I \backslash Q)$ and $\int_{Q}(1-h(s)) M(d s)$ are independent. Since the sequences $\left\{X_{n}^{\prime}\right\}$ and $\left\{X_{n}^{\prime \prime}\right\}$ are independent, we infer, by (4.6) and (4.7), that the random variables

$$
M(I \backslash Q)+\int_{Q}(1-h(s)) M(d s) \quad \text { and } \quad \int_{Q}(1-h(s)) M(d s)
$$

are independent. Hence it follows that $\int_{Q}(1-h(s)) M(d s)$ is a constant random variable. Finally, taking into account that the measure $M$ is isotropic, we infer that

$$
\int_{Q}(1-h(s)) M(d s)=0 .
$$

Consequently, by (4.7), $X_{0}^{\prime \prime}=M(I \backslash Q)$ and $X_{0}^{\prime}=M(Q)$. Setting $M_{1}(E)=M(E \cap Q)$ and $M_{2}(E)=M(E \cap(I \backslash Q))$, we have

$$
\begin{gathered}
X_{n}^{\prime}=T^{n} X_{0}^{\prime}=\int_{Q} e^{2 \pi n i s} M(d s)=\int_{I} e^{2 \pi n i s} M_{1}(d s), \\
X_{n}^{\prime \prime}=T^{n} X_{0}^{\prime \prime}=\int_{I \backslash Q} e^{2 \pi n i s} M(d s)=\int_{I} e^{2 \pi n i s} M_{2}(d s) .
\end{gathered}
$$

The theorem is thus proved.

We proceed now to a description of stationary harmonizable deterministic sequences $\left\{X_{n}(M)\right\}$ in terms of probabilistic characteristics of the random measure $M$. We remind that to every isotropic random measure $M$ there corresponds a Borel measure $\nu_{M}$ on $I$ and a function $\Phi_{M}$ on $I \times R_{+}$(see (3.4)). Moreover, by Theorem 3.1, the space $\mathscr{L}(M)$ is iden- tical with the Musielak-Orlicz space $L_{\Phi_{M}}\left(v_{M}\right)$. Further, the measure $v_{M}$ and the function $\Phi_{M}$ determine, by formula (1.4), a sequence of functions $\Omega_{\Phi_{M, v}, n}$ on $I$. It is evident that the sequence $\left\{X_{n}(M)\right\}$ is deterministic if and only if $X_{0}(M) \epsilon\left[X_{n}(M): n \leqslant-1\right]$. Since

$$
\left[X_{n}(M)\right]=\left\{\int_{I} f(s) M(d s): f \in \mathscr{L}(M)\right\},
$$

we infer, by virtue of Theorem 3.1, that $\left\{X_{n}(M)\right\}$ is deterministic if and only if

$$
\inf \left\|1+\sum_{k=1}^{n} a_{k} e^{-2 \pi k i t}\right\|=0,
$$

where the infimum is taken over all complex numbers $a_{1}, a_{2}, \ldots, a_{n}$ and $n=1,2, \ldots,\|\|$ being the norm in $L_{\Phi_{M}}\left(v_{M}\right)$. Since $\|f\|=\|\bar{f}\|$, we have, by Theorem 1.1, the following characterization of deterministic stationary harmonizable sequences:

THEOREM 4.3. A stationary sequence $\left\{X_{n}(M)\right\}$ is deterministic if and only if no function $\log \Omega_{\Phi_{M}, v_{M}, n}(n=1,2, \ldots)$ is Lebesgue integrable over $I$.

We say that $M$ is a Poisson random measure if there exist a probability distribution $P$ on $R^{2}$ and a non-negative Borel measure $\lambda$ on $I$ such that for every Borel subset $E$ of $I$ the probability distribution of $M(E)$ is given by the expression

$$
e^{-\lambda(E)} \sum_{n=0}^{\infty} \frac{\lambda^{n}(E) P^{* n}}{n !},
$$

where the power of $P$ is taken in the sense of convolution and $P^{* 0}$ denotes the probability measure concentrated at the origin. It is clear that the Poisson measure $M$ is isotropic if and only if the probability measure $P$ is isotropic. In this case the measure $\mu_{M}(E, \cdot)$ appearing in (3.1) is given by the formula

$$
\mu_{M}(E, A)=\lambda(E) \int_{A} \frac{t^{2}}{1+t^{2}} P_{0}(d t),
$$

where $P_{0}(A)=P(\{x:|x| \epsilon A\})$. Hence, by simple computations, it follows that the function $\Phi_{M}$ is bounded. Consequently, by definitions (1.3) and (1.4), the functions $\Omega_{\Phi_{M},{ }^{\nu}, n}$ are infinite almost everywhere. Thus from Theorem 4.3 we get the following

CoRollaRY. For every isotropic Poisson random measure $M$ the sequence $\left\{X_{n}(M)\right\}$ is deterministic.

Let $M$ be a Gaussian isotropic random measure. We have already mentioned that for Gaussian stationary sequences the concepts of predic- 
tion presented in this paper and the best linear least squares prediction coincide. It is easy to verify that for Gaussian isotropic measures $M$ the formula $\Phi_{M}(t, x)=\frac{1}{2} x^{2}$ is true. Consequently, $\mathscr{L}(M)=L^{2}\left(v_{M}\right)$ The classical characterization of completely non-deterministic wide sense stationary sequences (see [3], Chapter XII, §4) implies the following lemma:

LEMma 4.3. Let $M$ be a Gaussian isotropic random measure. The sequence $\left\{X_{n}(M)\right\}$ is completely non-deterministic if and only if either $M=0$ or the measure $\nu_{M}$ is absolutely continuous with respect to the Lebesgue measure and $\log \left(d \nu_{M} / d t\right)$ is Lebesgue integrable over $I$.

Now we shall give a description of stationary harmonizable completely non-deterministic sequences.

THEOREM 4.4. A stationary sequence $\left\{X_{n}(M)\right\}$ is completely now-deterministic if and only if either $M \equiv 0$ or $M$ is a Gaussian random measure, $v_{M}$ is absolutely continuous with respect to the Lebesgue measure and $\log \left(d v_{M} / d t\right)$ is Lebesgue integrable over $I$.

Proof. By Lemma 4.3, to prove the Theorem it suffices to prove that $M$ is a Gaussian random measure provided. $\left\{X_{n}(M)\right\}$ is stationary completely non-deterministic.

Let $A_{k}$ be the predictor based on the full past of $\left\{X_{n}(M)\right\}$ up to time $k$. Setting

$$
A_{k} X_{0}(M)=\int_{I} f_{k}(s) M(d s),
$$

where $f_{k} \in \mathscr{L}(M)$, and

we have

$$
E_{k}=\left\{s: f_{k}(s) \neq 1\right\}
$$

$$
A_{k} X_{0}(M)=\int_{\mathbb{E}_{k}} f_{k}(s) M(d s)+M\left(I \backslash E_{k}\right)
$$

Of course, the random variables $M\left(I \backslash E_{k}\right)$ and $\int_{n_{k}} f_{k}(s) M(d s)$ are independent and symmetrically distributed. Consequently, the relation

implies the relation

$$
\lim _{k \rightarrow-\infty} A_{k} X_{0}(M)=0
$$

$$
\lim _{k \rightarrow-\infty} M\left(I \backslash E_{k}\right)=0 .
$$

By the definition of predictors, the random variables $X_{0}(M)$ $-A_{k} X_{0}(M)$ and $X_{k}(M)$ are independent. In other words, the integrals

$$
\int_{I}\left(1-f_{k}(s)\right) M(d s) \text { and } \int_{I} e^{2 \pi k i s} M(d s)
$$

are independent. Since both integrands are different from 0 on $E_{k}$, we infer, by Theorem 2.1, that the random variable $M\left(E_{k}\right)$ is Gaussian.
Hence and from (4.8) it follows that $M(I)$, being the limit in probability of Gaussian random variables $M\left(E_{k}\right)$, is Gaussian too. By Cramér's Theorem ([10], p. 271), $M$ is a Gaussian random measure which completes the proof.

\section{References}

[1] S. Bernstein, Sur une propriété caractéristique de la loi de Gauss, Trans. Leningrad Polytech. Inst. 3 (1941), p. $21-22$

[2] G. Darmois, Sur une propriété caractéristique de la loi de probabilité de Laplace, C. R. Acad. Sci. Paris 232 (1951), p. $1999-2000$.

[3] J. L. Doob, Stochastic processes, New York 1953.

[4] N. D unford and J. T. Schwartz, Linear operators, Part I: General theory, New York 1958

[5] R. Gangolli, Abstract harmonic analysis and Lévy's Brownian motion of several parameters, Proceedings of the Fifth Berkeley Symposium on Mathematical Statistics and Probability, Vol. II, Part I (1967), p. 13-30.

[6] J. F. C. Kingman, Completely random measures, Pacifie Journal of Mathematics 21 (1967), p. $59-78$.

[7] A. N. Kolmogorov, Stationary sequences in Hilbert spaces (in Russian), Bull. MGU, Vol. II, No 6 (1941), p. I-40.

[8] M. G. Krein, On a generalization of some investigations of G. Szegö, V.I. Smirnov and A. N. Kolmogorov (in Russian), Doklady Acad. Sci. U. R. S. S. 46 (1945), p. $91-94$.

[9] R. G. Laha and E. Lukacs, On a characterization of the Wiener process, Transactions of the Second Prague Conference on Information Theory, Statistical Decision Functions and Random Processes, 1960, p. 307-312.

[10] M. Lò̀ve, Probability theory, New York 1950.

[11] W. Matuszewska, On generalized Orlicz spaces, Bull. Acad. Pol. Sci., Série Sci. Math., Astr. et Phys., 8 (1960), p. 349 -353.

[12] - Przestrzenie funkcji $\varphi: c a l l o w a l n y c h, ~ I$, Wlasności ogólne p-funkeji i klas funkcji p-calkowalnych, Prace Matematyczne 6 (1961), p. $121-139$.

[13] - and W. Orlicz, A note on the theory of s-normed spaces of $p$-integrable functions, Studia Math. 21 (1961), p. 107-115.

[14] J. Musielak and W. Orlicz, On modular spaces, ibidem 18 (1959), p. 49-65.

[15] A. Prékopa, On stochastic set functions I, Acta Math. Acad. Seient. Hung. 7 (1956), p. $215-263$.

[16] - On stochastic set functions II, ibidem, 8 (1957), p. 337 - 374.

[17] - On stochastic set functions III, ibidem 8 (1957), p. 375-400.

[18] V. P. Skitovich, On characterizing Brownian motion, Teor. Verojatnost.

i Primenen. 1 (1956), p. $361-364$.

[19] K. Urbanik, Prediction of strictly stationary sequences, Coll. Math. I? (1964), p. $115-129$.

[20] - Szegö's theorem for Orlicz spaces, Bull. Acad. Pol. Sci., Série Sci. Math., Astr. et Phys., 14 (1966), p. 503-509.

[21] - Some prediction problems for strictly stationary processes, Proceedings of the Fifth Berkeley Symposium on Mathematical Statistics and Probability, Vol. II, Part I (1967), p. $235-258$.

[22] - and W. A. Woyozyński, A random integral and Orlicz spaces, Bull. Acad. Pol. Sci., Ś́rie Sci. Math., Astr. et Phys., 15 (1967), p. 161-169. 
[23] H. Weyl, Über die Gleichverteilung von Zahlen mod. Eins, Matih. Annalen 77 (1916), p. $313-352$

[24] W. A. Woyczyński, Ind-additive functionals on random vectors, Disserta. tiones Mathematicae (to be printed).

[25] A. Zygmund, Trigonometrical series, Warszawa-Lwów, 1935.

INSTITUTE OF MATHEMATICS OF THE POLISH ACADEMY OF SOIENCES

INSTITUTE OF MATHEMATICS OF THE WROCHAW UNIVERSTTY

Reçu par la Rédaction le 9. 2. 1968

\section{Метод эквивалентных норм}

в теории абстрактных почти периодических функций

\section{М. И. КАДЕЩ (Харьков)}

Функция $x(t),-\infty<t<\infty$, со значениями в банаховом пространстве $E$ называется почти периодической (п. п. функцией), если она сильно непрерывна и если для каждого $\varepsilon>0$ можно указать такое $l=l(\varepsilon)$, что в любом интервале длины $l$ найдется хотя бы один $\varepsilon$-почти период ( $\varepsilon$-п. период) функции $x(t)$, то-есть число $\tau$ такое, что

$$
\sup _{t}\|x(t+\tau)-x(t)\|<\varepsilon \quad(-\infty<t<\infty) .
$$

Для числовых п. п. функций справедлива следующая теорема об интегрировании (см. [6], стр. 29):

Теорема Боля-Бора. Если интеграл

$$
X(t)=\int_{0}^{t} x(\eta) d \eta \quad(-\infty<t<\infty)
$$

n. n. функции $x(t)$ ограничен, то он также есть n. n. бункция. Более точно: Әля кажсдого $\varepsilon>0$ существует такое $\varepsilon_{1}=\varepsilon_{1}(x, \varepsilon)$, что каждый $\varepsilon_{1}-n$. период бункции $x(t)$ есть $\varepsilon-n$. период бункции $X(t)$.

Л. Америо [1], [2] показал, что теорема Боля-Бора распространяется на абстрактные п. п. функции, если в качестве $E$ взять равномерно выпуклое банахово пространство. Кроме того, он привел пример п. п. функции со значениями в пространстве $c$ всех сходящихся числовых последовательностей

$$
x(t)=\left\{\lambda_{n} \cos \lambda_{n} t\right\}_{n=1}^{\infty} \quad\left(\lambda_{n} \downarrow 0\right),
$$

интеграл от которой

$$
X(t)=\left\{\sin \lambda_{n} t\right\}_{n=1}^{\infty}
$$

есть ограниченная, но не почти периодическая, функция.

Естественно, возникает задача выделения тех пространств Банаха, которые, подобно равномерно выпуклым пространствам, допускают обобщение теоремы Боля-Бора. 